\title{
Interactive Analysis of Virtual Reality Technology in Residential Interior Design
}

\author{
Lili Zhu' a \\ ${ }^{1}$ Jiangxi Science \& Technology Normal University, Polytechnic Institute, Nanchang, Jiangxi, China, \\ 330038 \\ ${ }^{\mathrm{a}}$ email,
}

Keywords: Virtual Reality Technology; Interior Design; Residential

\begin{abstract}
With the continuous development of Chinese social economy, the study of virtual reality technology has become a hot topic in the academic field. Virtual reality technology has also been paid attention to by the community and gradually applied to people's lives. Based on the change and influence of the development of virtual reality technology in China, this paper further analyzes and summarizes the advantages of virtual reality technology in interior design application. And on this basis, the combination of interior design art and virtual reality technology gives the corresponding feasibility recommendations. With a view to Chinese contemporary interior design art to provide the corresponding innovative thinking, and promote the development of Chinese overall level of science and technology.
\end{abstract}

\section{Introduction}

Interior design activities are actually interior designers and users to communicate a process, carrying the designer's design philosophy and ideas in which. And Chinese interior design activities from the original hand-painted effect to the computer painting effect to the moment the animation effect, are inseparable from the use of technology. In the society, the combination of interior design activities and technological activities has become the trend of the development of the present art. Although the computer effect painting can show the designer's intention to a certain extent, the real effect of the modified program and computer painting renderings have been gradually difficult to meet the needs of Chinese current interior design activities, virtual technology in the use of interior design activities has become the new darling of people nowadays. However, from the current use of virtual reality technology in the design of residential interior design, virtual reality technology in Chinese popularity is not high, and this is because of people's awareness of virtual reality technology is not high, so this paper studies the interactive research of virtual reality technology in residential interior design, which plays an important role in promoting the development of interior design industry in China.

\section{The Status Quo of Industry}

From a global perspective, virtual reality technology is a high-tech preface of the scientific and technological means, through the further development of digital technology to extend an image virtual technology. The use of technology and research and development in the world are in a stage of exploration, but still cannot conceal its own obvious technical advantages and characteristics of light, to our various industries have brought a new technological revolution boom, so that people Experience the new interactive experience mode [1].

The use of virtual reality technology in Chinese use is also in an early stage, the construction industry because of its special industry nature, has introduced and applied the technology. In the early stage of the drawing, compared with the traditional hand-drawn drawings, more realistic, and the perfect show of the overall effect of early architectural design, greatly improving the efficiency of the architectural design industry, and from the design drawings effect, which is a kind of human-computer interaction, this interaction has been out of the traditional multimedia display means of computer, mouse and other manual operation to achieve a simulation of people and the 
environment for our virtual technology to provide the opportunity to communicate with the human body and interior design technology innovation. However, from the current situation of the use of virtual technology in Chinese current interior design, the use of relatively shallow, and the popularity of the country is not high, and people for the technology also has many concerns.

\section{The Feasibility of the Interactive Analysis of Virtual Reality Technology in Interior Design}

Therefore, the current use of virtual technology in real life, the impact of virtual technology for people is mainly reflected in the immersive and interactive.

The Reality of Virtual Reality Technology. The so-called immersion sense refers to the virtual reality technology through scientific and technological means to simulate the natural state, so that people in the visual and experience feel with a real feeling. The general virtual reality technology is the use of three-dimensional image forming technology for people to build a realistic environment with a similar virtual environment, and this feature of the virtual environment is the feeling that the sense of immersion. And when the virtual technology used in the interior design, people can according to their own imagination, will be projected to the corresponding screen. For example: the invention of a virtual helmet. This helmet allows people in the viewing room involved in the process of being in the process of isolation with the external visual auditory state, allowing users to better experience in the virtual scene, in order to achieve the final design of the program. This immersive feel is applied to the interior design, not only to help designers to improve their own design and perfect graphics will be displayed to the user, but also through the environment simulation, allowing users to enhance the design of the good sense, the user can also design according to the design details of the specific details of the adjustment, fully meet the user needs [2].

The Interaction of Virtual Reality Technology. In the use of virtual reality technology, the so-called interaction refers to the virtual environment under the influence of people on the degree of operation of things. The virtual reality technology applied to the interior design, through the use of hardware and software, in order to achieve the designer and the user to adjust the environment and other operations. By moving the scene, the 360 rotation space not only enhances the immersive sense of the design scheme, but also allows the user to enter the work of the work, which is based on the user's awareness enhancement, the synchronization presentation scheme modification effect and remodeling, the design scheme; Internal detailed observation.

\section{The Application Value of Virtual Reality Technology in Interior Design}

Create Immersive Interactive Design Activities. In today's information technology, intelligent technology has been widely used, modern interior design conforms to the trend of the times, accelerates the transformation of the interior design information, realizes the information exchange way as well as the information expression presentation, the traditional interior design in the process of the process and the process is too complicated, and its approach is too simple. And from the point of view of the effect of drawing graphics, the traditional renderings are difficult to express the designer's real ideas and design ideas, there is the user part of the design in a fuzzy understanding of the phenomenon, it is difficult to achieve the perfect communication between the user and the designer. The virtual reality technology has the immersion and interactive, perfect solution to meet the designers and user needs, filled the traditional interior design flaws, making the interactive design of the interior design possible. And based on the virtual reality technology of interior design, you can interactively transfer design information through the virtual environment to achieve a book design and technology combined with technology [3].

Improve the Efficiency of Interior Design Work. In the traditional interior design, the design resources mainly come from the designer's experience and the accumulation of cases, the existing information or cases according to user needs and the actual situation of the room to change accordingly to design a new design. But in the whole process, the need to spend a lot of designers of the labor force, and in the work cycle than long, there are many external factors, the work efficiency 
is relatively low. And based on the virtual reality technology under the interior design, can effectively design the decoration process, furniture, materials and other elements of digital words, and introduced into the interior design interactive analysis system. The use of flexible flexibility of virtual technology enhances the efficiency of the designer.

\section{Residential Interior Design Interactive Analysis System Construction Based on the Virtual Reality Technology}

Finish the Interior Design Data. Based on the principles of sustainable development and abide by the rules of interior design, we have collected the relevant data on the brand, size and price of the built-in residential interior space, furniture, household products, building materials, etc. from the shape, style and materials and the corresponding finishing induction, and finally entered into the interactive analysis system.

Establish 3D Model and Supporting Information Database. Virtual reality technology is the application of three-dimensional image, therefore, in the interior design process of interactive analysis system for the establishment of the 3D model and the corresponding supporting information database is essential. Based on the quantification of the interior design data (modeling, style, style, etc.) and the requirements of the variables (size, price, color, etc.), the three-dimensional information of the space is edited accordingly. The number of simulation of the three-dimensional effect of the graphics to provide interactive design can be used in the visual effects of the material parameters [4].

Develop Immersive Interactive Systems. The application of virtual reality technology, in the application of the current society there is a name - VH virtual reality technology, the technology to fully meet the user immersed and interactive on the basis of the designers and users to provide three-dimensional simulation environment, and use the current scientific and technological means to these simulation environment through the corresponding equipment projection, thus communication between users and design programs, designers communicate between the user bridge, real system to achieve the integration of interactive systems, and the system can pass sense of the virtual environment in the interaction, according to the quantitative requirements of the variable, real-time editing, in order to obtain the status of the user to meet.

\section{References}

[1] Li Gang. Application of Virtual Reality Technology in Architectural Interior Design[J / OL]. Technology Market, 2017, (07): 19-20.

[2] Xu Min. Virtual reality technology in the interior design application of skilled personnel training research[J]. Art Technology, 2017, 30 (04): $21+23$.

[3] Cao Jin, Tai Jie, Zhou Bei.Discussion on the Application of Virtual Reality Technology in Interior Space Design [J / OL]. Design, 2017, (05): 26-27.

[4] Xiong Xuejiao.Application of virtual reality technology in interactive analysis of residential interior design[J]. Modern Decoration (Theory), 2017, (01): 17. 